\title{
Robust Detection of the North-West African Upwelling From SST Images
}

\author{
Anass El Aouni ${ }^{\circledR}$, Khalid Daoudi, Khalid Minaoui, and Hussein Yahia ${ }^{\circledR}$
}

\begin{abstract}
Analysis and study of coastal upwelling using sea surface temperature (SST) satellite images is a common procedure because of its coast effectiveness (economic, time, frequency, and manpower). Developing on the Ekman theory, we propose a robust method to identify the upwelling regions along the northwest African margin. The proposed method comes to overcome the issues encountered in a recent method devoted for the same purpose and for the same upwelling system. Afterward, we show how our method can serve as a framework to study and monitor the spatio-temporal variability of the upwelling phenomenon in the studied region.
\end{abstract}

Index Terms-Sea surface temperature (SST) images, upwelling, upwelling identification and extraction, upwelling index.

\section{INTRODUCTION}

$\mathbf{T}$ HE Atlantic coastline of north Africa is characterized by the presence of the upwelling phenomenon almost all around the year. This phenomenon can be identified by seasonally variable cold and nutrient-rich water [1]. Thus, the automatic identification and detection of this phenomenon is of fundamental importance in the study of productivity enhancement [2], [3] and associated fish management [4].

In the literature, several techniques and algorithms have addressed the problem of automated identification of upwelling regions in sea surface temperature (SST) images. For instance, Cayula and Cornillon [5] proposed an unsupervised classification method where neural network is trained based on the $k$-means clustering [6], [7]. Nieto et al. [8] proposed a histogram-based separation method, where the bimodality of SST histogram is interpreted to represent two different water. Sousa et al. [9] proposed an algorithm to extract upwelling regions in the coastal ocean of Portugal. This algorithm is based on fuzzy clustering and yields good segmentation accuracy in that particular area. More recently, Tamim et al. and Anass El Aouni et al. [10]-[12] improved the latter algorithm and developed a method that showed good results in the southern part of the Moroccan Atlantic coast. However, this method fails to provide good segmentation on the whole coast of Morocco (including the central and

Manuscript received January 13, 2020; accepted March 25, 2020. This work was supported by the French-Moroccan Partenariats Hubert Curien (PHC)-Toubkal under Project TBK/16-24 and Project PPR2-6. (Corresponding author: Anass El Aouni.)

Anass El Aouni, Khalid Daoudi, and Hussein Yahia are with INRIA Bordeaux Sud-Ouest, 33405 Talence, France (e-mail: anas.elaouni@gmail.com; khalid.daoudi@inria.fr; hussein.yahia@inria.fr).

Khalid Minaoui is with the Faculty of Sciences, Mohammed V University in Rabat, LRIT URAC 29, Rabat 10004, Morocco (e-mail: khalid.minaoui@um5.ac.ma).

Color versions of one or more of the figures in this letter are available online at http://ieeexplore.ieee.org.

Digital Object Identifier 10.1109/LGRS.2020.2983826 northern regions). This is mainly due to the high variability of the latitudinal distribution of temperatures.

In this letter, we develop a robust approach devoted to the identification of the upwelling regions from SST images along the central part of the Canary upwelling Ecosystem. Our method is based on a nonlinear normalization of SST images, it is simple to implement and it overcomes the problems encountered while using the method developed in [10] and [11]. Also in this letter, we show how our method can serve as a framework to monitor the spatio-temporal variability of the upwelling intensity and extent.

The letter is structured as follows: Section II presents the database and the area of study used throughout this letter. Section III describes the methodology for the automatic delimitation of upwelling area from SST images. In Section IV, we show how our method can serve as an input to characterize and monitor the evolution of the upwelling variability in the region. Finally, a conclusion is drawn in Section V.

\section{REgion OF Interest AND DATA SETS}

In this letter, we use a total of 720 thermal level 3 infrared MODIS-T images of the Moroccan Atlantic coast spanning the region $20^{\circ} 510-36^{\circ} 21 \mathrm{~N}$ and $4^{\circ} 26-19^{\circ} 97 \mathrm{~W}$, for the time period from January 2001 to December 2015. These data are extracted from http://oceancolor.gsfc.nasa.gov/ a with spatial resolution of $4 \times 4 \mathrm{~km}$. Fig. 1(a) shows an example of SST images over the study area, obtained on the first week of 2007. We consider eight-day SST images because they constitute a good compromise between the variability of the pixels values and the necessity to provide a continuous spatio-temporal coverage. SST images are included in the database if they have at least $25 \%$ of valid information in the area between the coast and $200 \mathrm{~km}$ offshore.

\section{Segmentation of Upwelling AReas}

In this section, we present a robust method to extract the upwelling regions along the Moroccan Atlantic coast, this method complements and improves the method introduced in [10] and [11]. Indeed, the latter's region of interest is the south part of Morocco. Although it yields accurate segmentation in that region, it fails however, to provide good segmentation on the whole coast of Morocco (including center and north). The main reason behind this failure is the high variability of the latitudinal distribution of temperatures. Hence, for example, upwelling temperature in the southern part can be closer to the offshore temperature in the northern region. An example of such phenomena is shown in Fig. 1. In this figure, the green curve represents the latitudinal variation of 


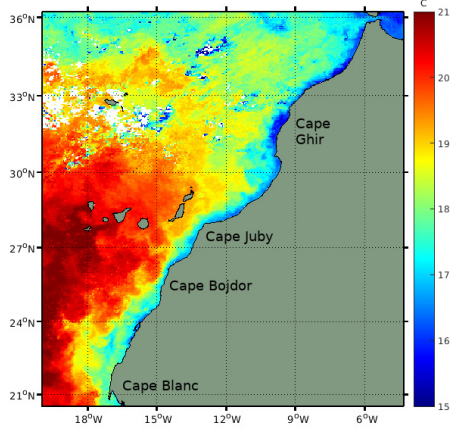

(a)

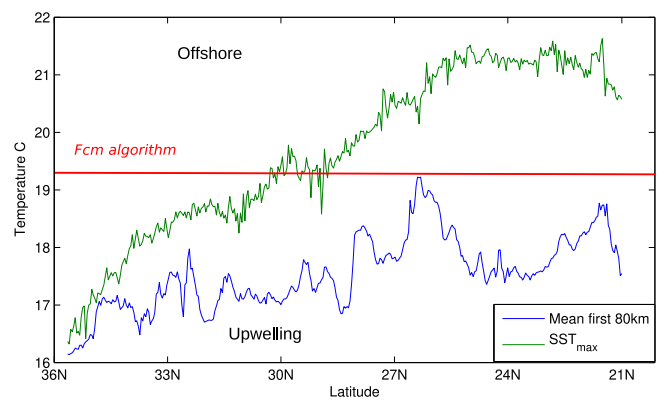

(c)

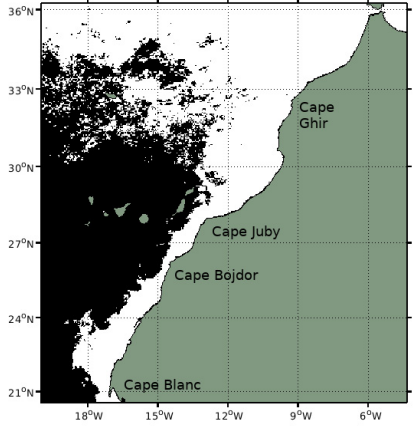

(b) (a) Eight-day SST images obtained during the first week of January 2007. (b) Result of the approach developed in [11] applied on the SST image from (a). White color refers to upwelling water, whereas black color refers to offshore water. (c) Latitudinal comparison between the upwelling and the offshore temperature; green curve represents the maximal temperature on each latitudinal step of the SST image in (a), blue curve represents the mean temperature over the first $80 \mathrm{~km}$ of each latitudinal step.

the maximal temperature in the offshore along the SST image in Fig. 1(a). The blue curve represents the latitudinal variation of the mean temperature over the first $80 \mathrm{~km}$. Here, the red line represents the Fuzzy C-means algorithm (FCM), which separates the two clusters (upwelling and nonupwelling), and this figure shows that a part of the green curve falls in the cluster presenting the upwelling water. To bypass this problem, we propose a latitudinal normalization by offshore temperatures, that is, we consider "region-dependent" difference of temperatures rather the temperatures themselves. The procedure is described in Section III-A.

\section{A. Latitudinal Normalization of SST Images}

As described in [13], Ekman transport can generate a $90^{\circ}$ movement of surface water to the right of the wind direction, which results in coastal upwelling in the case of the north-west African margin. Thus, in order to identify the upwelling, it is natural to compare the temperature of the inshore water with the offshore water within a perpendicular line to the coast. Within each perpendicular line, apply the method developed in [10] and [11] and find what pixels belong to the upwelling. This turns out to be similar to normalizing every line with respect to its maximum temperature found in the offshore. In this letter, we thus partition each image into $r$ lines $l$ which are perpendicular to the coast, as shown in Fig. 2, where $l$ are pixel of width and $n$ pixels of length starting from the coast line. All the lines present the same angle to the coast line in order to cover all the pixels of the image as follows:

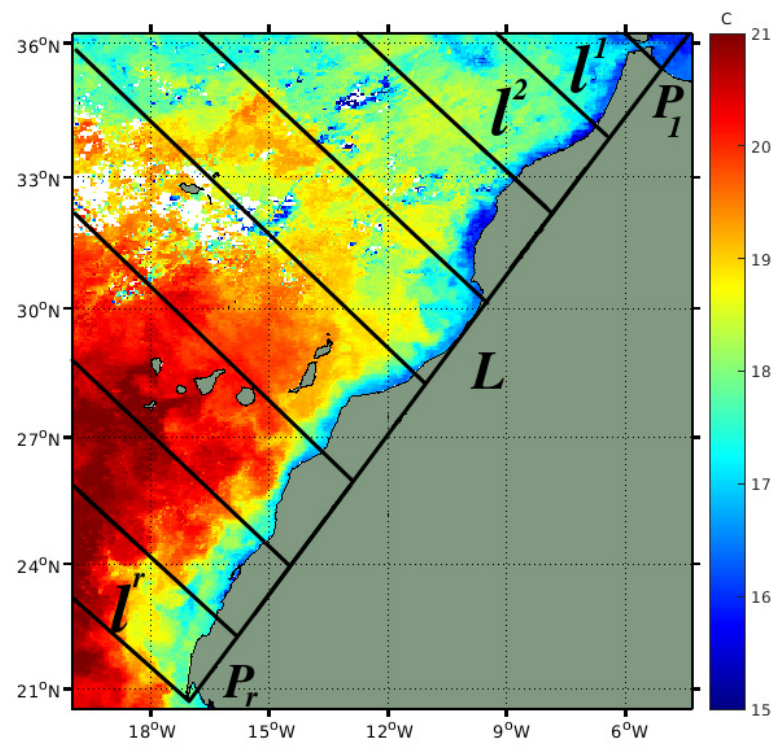

Fig. 2. Illustration of the nonlinear normalization of SST images. $L$ represents the general orientation of the Moroccan coast, $l^{1}, \ldots, l^{r}$ represents the perpendicular lines to the coastline.

We first define the general orientation of the Moroccan coast as a virtual line

$$
L_{\text {lat }, \text { lon }}=\left(P_{1}, \ldots, P_{r}\right) .
$$

Starting from the northern point $P_{1}$ till the southern one $P_{r}$. This line will serve to define the perpendicular lines as

$$
\begin{gathered}
\left\langle l^{1}, L\right\rangle=0 \\
\vdots \\
\left\langle l^{r}, L\right\rangle=0 .
\end{gathered}
$$

On each line, we first define the maximal offshore temperature and normalize the line with respect to its maximum found in the offshore up to the limit of the image boundary. To do so, we consider a line $l \in\left\{l_{1}, \ldots, l_{r}\right\}$ composed of temperature pixels $l=\left\{T_{i}^{n} ; i=1, \ldots, n\right\}$. Let $T_{m}^{l}$ be the maximal temperature on line $l$

$$
T_{m}^{l}=\max _{i} T_{i}^{l} .
$$

We now define the offshore difference of temperature as

$$
\delta T_{i}^{l}=T_{m}^{l}-T_{i}^{l} .
$$

Fig. 3(a) displays the image of Fig. 1(a) after this normalization. As we see, this image keeps the same thermal structures as in the SST image. Moreover, the upwelling area, which now corresponds to high pixel intensities, is visually more clear than in the original SST image. However, there exists some unnatural lines caused by the fluctuations presented in the vector of maximal temperatures $T_{m}^{r}$ [red line in Fig. 3(c)] used to generate this image. To overcome this problem, a moving average algorithm with a window size of five is used to smooth out the vector of maximal temperatures [blue line in Fig. 3(c)]. Fig. 3(b) shows the final normalized image computed using the smoothed $T_{m}^{r}$ vector.

Fig. 4 displays the "dual" information of Fig. 1 where the green curve represents the minimal pixel in each line of the 


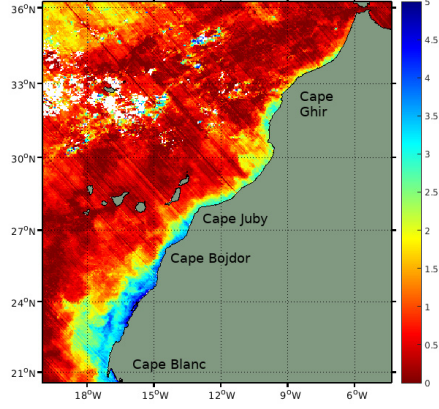

(a)

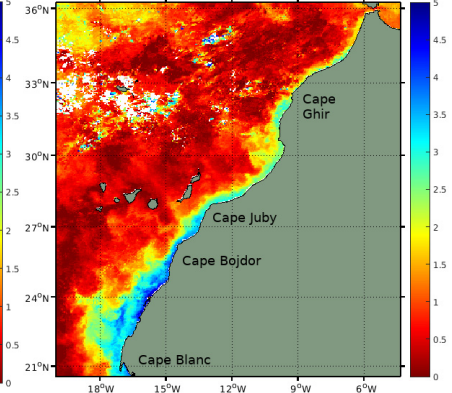

(b)

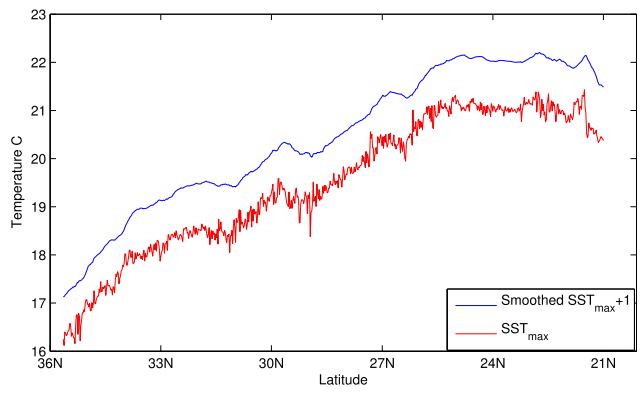

(c)

Fig. 3. (a) Normalized SST image computed from the SST images in (a). (b) Smoothed normalized SST image. (c) $\mathrm{SST}_{\max }$ vector before (red) and after (blue) being smoothed (increased by 1).

normalized image and the blue curve represents the mean pixels over the first $80 \mathrm{~km}$ of each line. It is clear that offshore information (green curve) is now normalized over the whole coast and is clearly discriminated from the inshore information (blue curve).

\section{B. Upwelling Segmentation}

Once the normalization is applied on an SST image, the upwelling area corresponds to the region(s) with the highest intensities. Segmentation of this area goes back to separate the latter from pixels with the lowest intensities. To do so, we use the same approach developed in [11] and [10] but applied over the normalized image this time.

Fig. 1(a) displays the segmentation obtained with the method developed in [11] applied on the SST images in Fig. 1(a). As stated above, this method fails to provide a good segmentation along the whole coast. Fig. 4(a) shows the segmentation obtained after applying the same method on the normalized SST images. It is so clear that the new approach yields a very accurate segmentation on the whole coast, therefore, solved the problem encountered in the northern parts of the upwelling system.

\section{FRAMEWORK FOR UPWELLING MONITORING}

This section shows how the proposed method can be used as an input to study and monitor the evolution of the upwelling phenomenon along the studied area. Several authors have used SST images to characterize and quantify the spatio-temporal variability of the upwelling intensity, generally calculated as a simple thermal difference between the inshore water and its

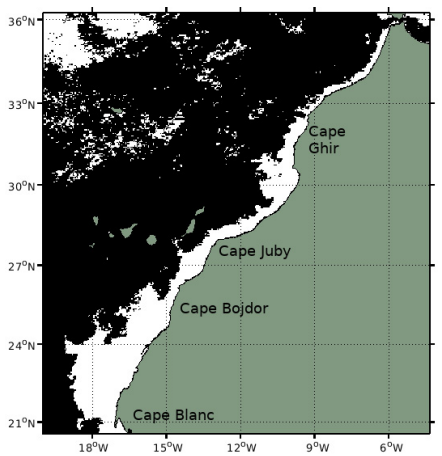

(a)

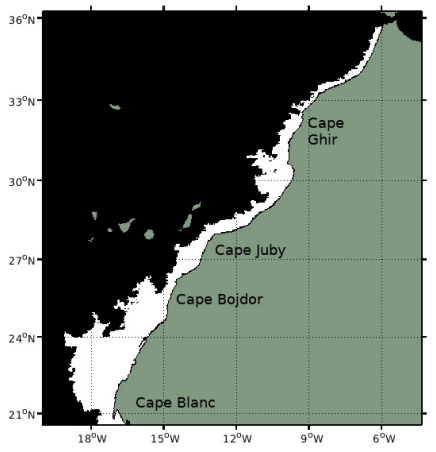

(b)

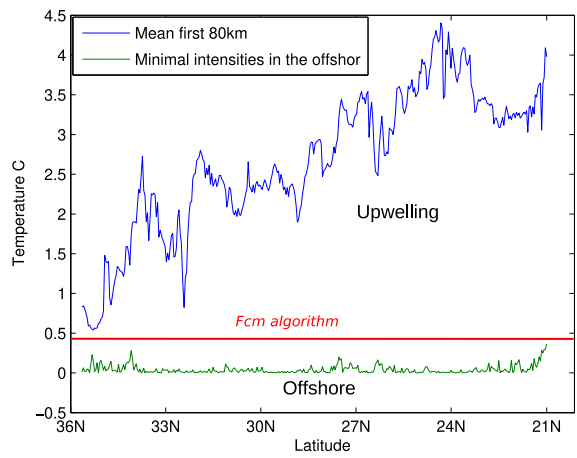

(c)

Fig. 4. (a) Binary result of FCM algorithm applied on the normalized SST image in (b). White color refers to upwelling water while black color refers to offshore water. (b) Binary result after the application of the regiongrowing algorithm [14] on the image (a). (c) Latitudinal comparison between the upwelling and the offshore pixels values.

reference in the offshore direction [15]-[19]

$$
I^{l}=T_{\max }^{l}-T_{\min }^{l} .
$$

In the literature, $T_{\min }^{l}$ is defined as the minimum temperature recorded in the coastal band from the coast up to the continental slope [15]-[18]. For the Moroccan coast, the latter is generally chosen to be $500 \mathrm{~km}$.

Here, we simply define $T_{\min }^{l}$ as the minimal temperature encountered within the extracted upwelling region as shown in Fig. 5. That is, let $S$ be the extracted upwelling region, then given a line $l \in\left\{l_{1}, \ldots, l_{r}\right\}$ of temperature pixels $l=\left\{T_{i}^{l} ; i=\right.$ $1, \ldots, n\}, T_{\min }^{l}$ is defined as

$$
T_{\min }^{l}=\min _{i \in S} T_{i}^{l}
$$

This approach is simple, very intuitive, and dynamic because it takes into account the spatial extent of the upwelling, therefore, it ensures that all the (necessary) low temperatures are considered in the calculation. We show in Fig. 6 the interannual variability of the upwelling intensity via a space-time Hovmoller diagram computed from the definition (5 and 6) at $4 \mathrm{~km} / 8$-day spatio-temporal resolution. The upwelling intensity displays high variability both in space and time, with the entire period being marked by substantial oscillations between strong and weak intensity, and a main upwelling pattern that can be divided into three characteristic regions. 


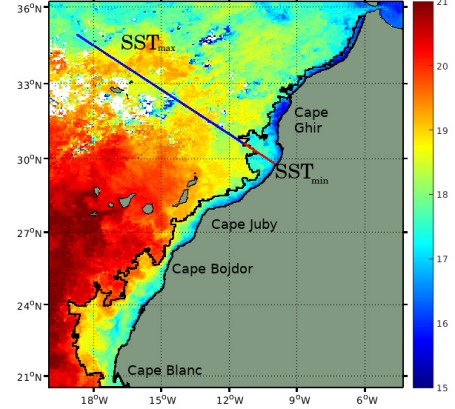

(a)

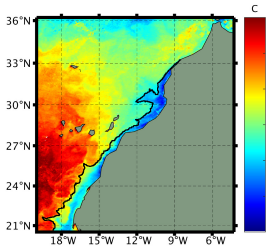

(b)

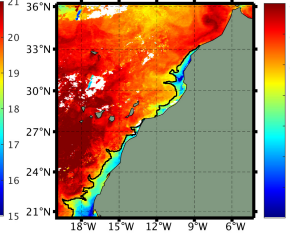

(c)

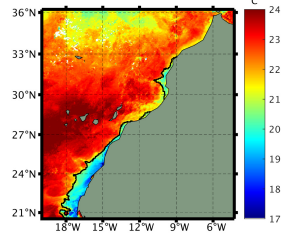

(d)
Fig. 5. (a) Final segmentation achieved by the proposed method. Also shown in the image, an example of a line within which the coastal upwelling $I^{l}$ index is computed. (b)-(d) shows the results of the proposed method applied on different eight-day images covering different periods of time (April, June, and September).

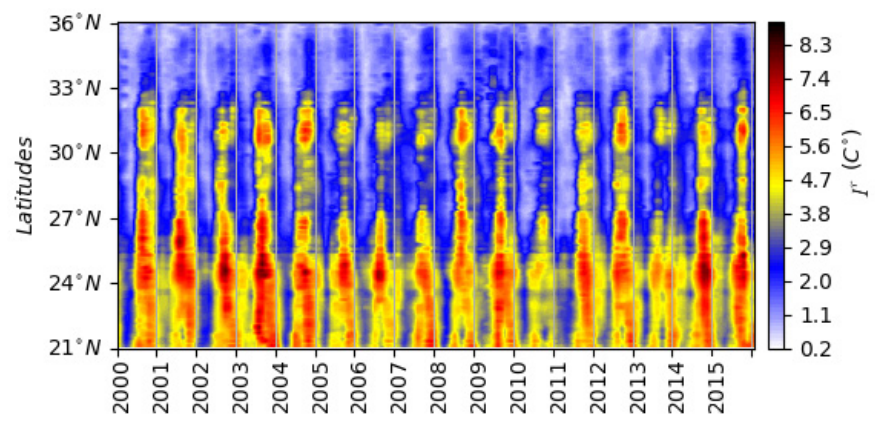

Fig. 6. Space-time Hovmoller plot of the interannual variability of the upwelling intensity $I^{l}$ computed from the definition (5) and (6) from 2001 to 2015

1) The region between $33^{\circ} \mathrm{N}$ and $36^{\circ} \mathrm{N}$ where the upwelling intensity is weak due to the unfavorable orientation of the coast line in this region.

2) The region between $27^{\circ} \mathrm{N}$ and $33^{\circ} \mathrm{N}$, where the upwelling intensity is strong in summer and autumn.

3) The region between $21^{\circ} \mathrm{N}$ and $27^{\circ} \mathrm{N}$ where the upwelling is intense throughout the year with a maximum from spring to autumn.

\section{CONCLUSION}

This letter contributes to the study and monitoring of the upwelling phenomenon over the north-west African margin. Previous works have addressed the issue of the automatic identification of upwelling regions from the SST images along different parts of the Canary current upwelling system. However, the problem of upwelling extraction from SST images along the central part of the Canary Current upwelling system (CCUS) shows several challenges in the literature. In this letter, an algorithm that successfully delineates the upwelling regions from the SST images over the whole central part of CCUS is proposed. The proposed approach is performed in two steps: the first one where a nonlinear normalization is applied over each SST image, and the second where a clustering algorithm is applied over the normalized SST images to separate the region of upwelling from the offshore water. This method showed to be very accurate and more robust than a recent state-of-the-art method. Moreover, the proposed method can be served as a framework to study and monitor the spatio-temporal variability of the upwelling dynamics over the studied area.

\section{REFERENCES}

[1] A. El Aouni, V. Garçon, J. Sudre, H. Yahia, K. Daoudi, and K. Minaoui, "Physical and biological satellite observations of the northwest African upwelling: Spatial extent and dynamics," IEEE Trans. Geosci. Remote Sens., vol. 58, no. 2, pp. 1409-1421, Feb. 2020.

[2] A. El Aouni, K. Daoudi, H. Yahia, K. Minaoui, and A. Benazzouz, "Surface mixing and biological activity in the north-west African upwelling," Chaos, Interdiscipl. J. Nonlinear Sci., vol. 29, no. 1, Jan. 2019, Art. no. 011104

[3] A. El. Aouni, K. Daoudi, H. Yahia, and K. Minaoui, "The contribution and influence of coherent mesoscale eddies off the North-West African Upwelling on the open ocean," in Proc. SIAM Conf. Math. Planet Earth (MPE), Philadelphia, PA, USA, Sep. 2018.

[4] E. Chassot et al., "Satellite remote sensing for an ecosystem approach to fisheries management," J. Mar. Sci., J. Conseil, vol. 68, no. 4, pp. 651-666, 2011.

[5] J.-F. Cayula and P. Cornillon, "Edge detection algorithm for SST images," J. Atmos. Ocean. Technol., vol. 9, no. 1, pp. 67-80, Feb. 1992.

[6] J. A. Hartigan and M. A. Wong, "Algorithm AS 136: A K-means clustering algorithm,” J. Roy. Stat. Soc. C, Appl. Statist., vol. 28, no. 1, pp. 100-108, 1979.

[7] A. Likas, N. Vlassis, and J. J. Verbeek, "The global K-means clustering algorithm," Pattern Recognit., vol. 36, no. 2, pp. 451-461, Feb. 2003.

[8] K. Nieto, H. Demarcq, and S. McClatchie, "Mesoscale frontal structures in the canary upwelling system: New front and filament detection algorithms applied to spatial and temporal patterns," Remote Sens. Environ., vol. 123, pp. 339-346, Aug. 2012.

[9] F. M. Sousa, S. Nascimento, H. Casimiro, and D. Boutov, "Identification of upwelling areas on sea surface temperature images using fuzzy clustering," Remote Sens. Environ., vol. 112, no. 6, pp. 2817-2823, Jun. 2008.

[10] A. Tamim, K. Minaoui, K. Daoudi, H. Yahia, A. Atillah, and D. Aboutajdine, "An efficient tool for automatic delimitation of moroccan coastal upwelling using SST images," IEEE Geosci. Remote Sens. Lett., vol. 12, no. 4, pp. 875-879, Apr. 2015.

[11] A. Tamim et al., "A simple and efficient approach for coarse segmentation of Moroccan coastal upwelling," in Proc. 21st Eur. Signal Process. Conf. (EUSIPCO), 2013, pp. 1-5.

[12] A. El Aouni, K. Minaoui, A. Tamim, K. Daoudi, and H. Yahia, "An improved method for accurate computation of coastal upwelling index using sea surface temperature images," in Proc. 9th Int. Symp. Signal, Image, Video Commun. (ISIVC), Nov. 2018, pp. 76-81.

[13] V. W. Ekman, "On the influence of the Earth's rotation on oceancurrents," Ark. Mat. Astron. Fys., vol. 2, pp. 1-53, 1905.

[14] Y.-L. Chang and X. Li, "Adaptive image region-growing," IEEE Trans. Image Process., vol. 3, no. 6, pp. 868-872, Nov. 1994.

[15] L. Van Camp, L. Nykjaer, E. Mittelstaedt, and P. Schlittenhardt, "Upwelling and boundary circulation off northwest Africa as depicted by infrared and visible satellite observations," Prog. Oceanogr., vol. 26, no. 4, pp. 357-402, Jan. 1991.

[16] L. Nykjær and L. Van Camp, "Seasonal and interannual variability of coastal upwelling along northwest Africa and Portugal from 1981 to 1991," J. Geophys. Res., vol. 99, no. C7, pp. 14197-14207, 1994.

[17] H. Demarcq and V. Faure, "Coastal upwelling and associated retention indices derived from satellite SST. Application to octopus vulgaris recruitment," Oceanologica Acta, vol. 23, no. 4, pp. 391-408, Aug. 2000.

[18] A. M. P. Santos, A. S. Kazmin, and A. Peliz, "Decadal changes in the Canary upwelling system as revealed by satellite observations: Their impact on productivity," J. Mar. Res., vol. 63, no. 2, pp. 359-379, 2005.

[19] J. Marcello, A. Hernández-Guerra, F. Eugenio, and A. Fonte, "Seasonal and temporal study of the northwest African upwelling system," Int. J. Remote Sens., vol. 32, no. 7, pp. 1843-1859, Mar. 2011. 